\title{
Linear current module adaptive to wide operating voltage range based on automatic series/parallel conversion
}

\author{
Kui Ma ${ }^{1, \text { a) }}$, Zhuang Wang ${ }^{1}$, and Fashun Yang ${ }^{2}$
}

Abstract A new method based on adaptive series/parallel conversion to realize linear current output in a wide operating voltage range is presented in this paper. According to the load state, a switch array is employed to perform adaptive series/parallel conversion control on load segments and corresponding constant current branches to ensure constant current in each load segment and constant power of the total load in wide operating voltage range. The proposed design combines the advantages of switch mode current source and linear constant current source. The correctness and feasibility of the proposed method are verified by experiments of a LED driver.

Keywords: linear current, constant current sources, adaptive control, wide operating voltage range, LED driver

Classification: Circuits and modules for electronic instrumentation

\section{Introduction}

Current source, which includes switch mode and linear mode, is widely used in electric appliances, communications facilities, portable devices, etc. [1, 2, 3, 4, 5, 6, 7, 8, 9, 10, 11]. In switch mode, power devices work in on-off state with the control of a high frequency $(\sim \mathrm{kHz}$ or $\sim \mathrm{MHz})$ signal $[4,10,12,13,14,15,16,17,18,19]$. So, the switch mode current source facilitates high conversion efficiency and small volume. However, ripple, electromagnetic interference (EMI) and relatively complex control circuit are drawbacks of switching power supply $[18,19]$. Whereas, in linear mode, power devices usually work in constant current state with the control of feedback signal [20, 21, 22, 23, 24]. The advantages of linear current source are small ripple of output current, high accuracy of constant current and simple circuit, etc. But the continuous conduction of power devices will result in low conversion efficiency [8].

In this paper, combining the advantages of switch mode and linear mode current sources [25], a novel linear current module with wide operating voltage range based on adaptive series/parallel conversion is proposed. As the operating voltage changes from low to high, the connection of load branches changes from parallel to hybrid, and finally to series. And the number of parallel constant current branches is correspondingly gradually increasing. The current in each

${ }^{1}$ Dept. of Electronics, Guizhou University, Guiyang 550025, China

${ }^{2}$ Semiconductor Power Device Reliability Engineering Research Center of Ministry of Education, Guiyang 550025, China

a) kma@gzu.edu.cn

DOI: $10.1587 /$ elex.17.20200191

Received May 27, 2020

Accepted June 16, 2020

Publicized June 26, 2020

Copyedited July 10, 2020 load branch is constant. And the power of total load is constant, too. The adaptive conversion of connection of current branches and load branches is controlled by a switch array. LED driver is one of the main applications of constant current source [23, 24, 26, 27, 28, 29, 30, 31]. Based on the proposed linear current module, a four-segment LED driver is designed and experimentally verified. Four LED segments and four constant current branches are connected in parallel, respectively, and constant current connects LED block in series at lower operating voltage. At middle operating voltage, two parallel constant current branches connect two parallel LED branches in series. Two LED segments connect in series in each LED branch. And a constant current branch connects four series LED segments in series at higher operating voltage. During the whole operating voltage range $(15-60 \mathrm{~V})$, the current in each LED segment is nearly the same and stable, and the total power of LEDs is invariable.

\section{Design and analysis}

Conventionally, switch mode current sources provide relatively large current to load. Several branches of load driven by a switch mode current source are connected in parallel. The output current of linear constant current modules is always relatively lower. Loads driven by a linear constant current source are always connected in series. The voltage drop on the linear constant current module will change greatly due to the wide range of operating voltage. In order to reduce the variation of voltage drop on the linear constant current module, the connection between constant current branches and load branches can be adaptively changed from parallel to hybrid, and finally to series as the operating voltage changes from low to high.

The block diagram of the proposed linear current module adaptive to wide operating voltage range is shown in the dashed box in Fig. 1. In this module, constant current block and load are connected in series. The linear current block is averagely divided into $n$ branches ( $n$ is even number). And correspondingly, load is averagely divided into $n$ segments. According to the controlling vector $E N \_1, E N \_2$, to $E N \_n$ from the comparison of voltage reference $V_{\text {ref_1 }}$ to $V_{\text {ref_m }}$ and the detection of the input DC voltage $V_{\mathrm{d}}$, the switch array controls $n$ load segments connect in series, hybrid and parallel, and the number of constant current branches correspondingly increase according to the number of load branches in parallel, during the operating voltage changes from low to high. When the input DC operating voltage is lower (as- 


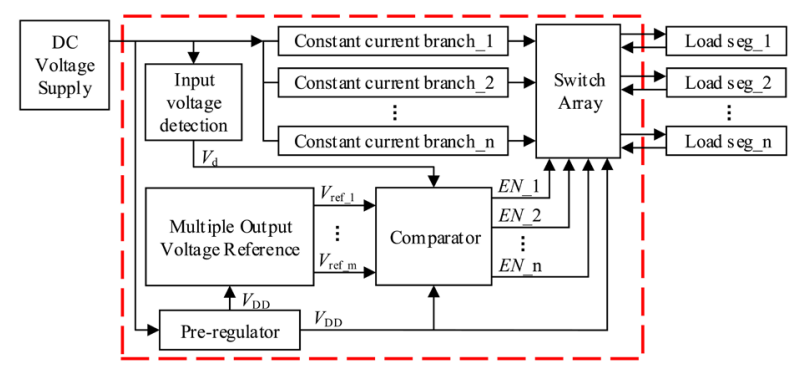

Fig. 1 Block diagram of linear current module adaptive to wide operating voltage range.

suming the threshold voltage is $\left.V_{\mathrm{T} 1}, V_{\mathrm{DC}}<V_{\mathrm{T} 1}\right)$ all constant current branches and all load segments are connected in parallel, respectively. And then the constant current block and the load are connected in series. Setting this mode as parallel mode.

As the input DC operating voltage rises, the switch array adaptively parallels appropriate constant current branches and correspondingly transfers the load into hybrid connection. With the increase of DC operating voltage, the number of segments connected in series is gradually increased and the number of parallel load branches is correspondingly gradually reduced. The number of connected constant current branches in parallel is consistent with the number of parallel branches in the load of hybrid connection. Setting this mode as hybrid mode. Taking $n=12$ for example, five threshold voltages $\left(V_{\mathrm{T} 1}, V_{\mathrm{T} 2}, V_{\mathrm{T} 3}, V_{\mathrm{T} 4}, V_{\mathrm{T} 5}\right)$ are needed. setting $a$ is the number of load branches, $b$ is the number of segments connected in series in a load branch, and $c$ is the number of constant current branches in parallel. In $h y-$ brid mode, when $V_{\mathrm{T} 1}<V_{\mathrm{DC}}<V_{\mathrm{T} 2}, a=6, b=2$, and $c=6$. When $V_{\mathrm{T} 2}<V_{\mathrm{DC}}<V_{\mathrm{T} 3}, a=4, b=3$, and $c=4$. When $V_{\mathrm{T} 3}<V_{\mathrm{DC}}<V_{\mathrm{T} 4}, a=3, b=4$, and $c=3$. When $V_{\mathrm{T} 4}<V_{\mathrm{DC}}<V_{\mathrm{T} 5}, a=2, b=6$, and $c=2$.

While the input DC operating voltage is high enough (assuming the threshold voltage is $V_{\mathrm{Tm}}, V_{\mathrm{DC}}>V_{\mathrm{Tm}}$ ), a constant current branch is connected in series with all series connected load segments. Setting this mode as series mode.

The design described above is verified by a LED linear current driver. In linear constant current drivers of LED, segmented linear constant current source, such as lighting LED segments up one by one as the operating voltage goes from low to high $[28,29,30]$, or turning on LEDs on the opposite arm of a rectifier bridge composed of LEDs in each half cycle of the city electricity [31], is a driving method with obvious advantages in voltage operating range, conversion efficiency, etc.. However, at present, the significant drawback of commercial segmented linear constant current driving methods of LED is that the lighting of LEDs is not stable within the whole operating voltage range.

Fig. 2 shows the LED driving circuit based on the proposed linear current module adaptive to wide operating voltage. The constant current block is composed by four branches. A constant current branch is a $20 \mathrm{~mA}$ current regulative diode (CRD). The LED load are divided into four segments. In each segment, there are three LEDs. The power of a LED is $3.2 \mathrm{~V} \times 20 \mathrm{~mA}$. During the full range of the DC power voltage $(15-60 \mathrm{~V})$, the detection voltage $V_{\mathrm{d}}$

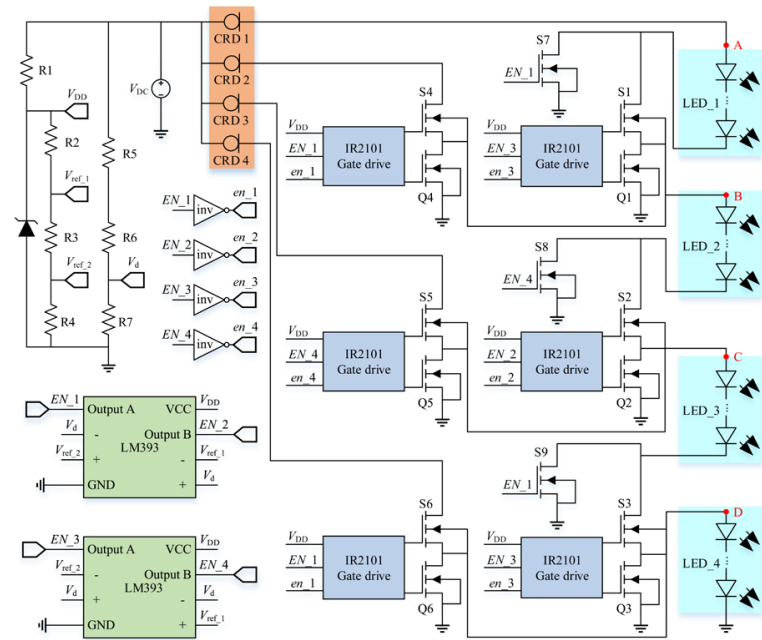

Fig. 2 LED linear current driving circuit based on adaptive series/parallel conversion for wide operating voltage range.

obtained by resistance voltage divider is compared with two voltage references $V_{\text {ref_1 }}$ and $V_{\text {ref_2 }}$ obtained by a simple circuit based on Zener diode to generate control signal $E N \_1$, $E N \_2, E N \_3$ and $E N \_4$ to control the status of switches in switch array. Under low DC power voltage $(15 \mathrm{~V}-24 \mathrm{~V})$, switches S1, S2 and S3 are off, and others are on. Four parallel connected constant current branches provide $80 \mathrm{~mA}$ current to drive four parallel connected LED segments. As the DC power voltage rises to $24 \mathrm{~V}-48 \mathrm{~V}$, switches S1, S3, S5 and $\mathrm{S} 8$ are on, and others are off. Two parallel connected constant current branches provide $40 \mathrm{~mA}$ current to drive two parallel connected load branches. In each load branch, two LED segments connect in series. While the DC power voltage is in $48 \mathrm{~V}-60 \mathrm{~V}$, switches S1, S2 and S3 are on, and others are off. One constant current branch provides $20 \mathrm{~mA}$ current to drive four series connected LED segments. MOSFETs Q1-Q6 correspondingly provide floating control for S1-S6, respectively.

\section{Experimental verification}

In the experimental circuit, constant current voltage range of CRDs is 4.5-40V. Setting $V_{\text {ref_1 }}=4 \mathrm{~V}, V_{\text {ref_2 }}=2 \mathrm{~V}, \mathrm{R} 1=$ $1 \mathrm{k} \Omega$, R2: R3: R4 = 3: 1: 1, R5: R6: R7 = 10: 1: 1. Testing results show that $V_{\mathrm{DD}}$ varies from $10.18 \mathrm{~V}$ to $10.91 \mathrm{~V}$ for $V_{\mathrm{DC}}$ in the range of $15-60 \mathrm{~V}$. Power supply voltage regulation rate of $V_{\mathrm{DD}}$ is $16.2 \mathrm{mV} / \mathrm{V}$.

When $V_{\mathrm{DC}}$ varies in the range of $15-60 \mathrm{~V}$, tested results of control vector, state of switches, connection mode of LED segments, and voltage drop on each LED segment are given in Table I. For $V_{\mathrm{DC}}$ in $15-24 \mathrm{~V}, E N \_1=E N \_2=$ 1, EN_3 = EN_4 =0, switches S4, S5, S6, S7, S8, S9 are turned on to control four LED segments connected in parallel. $V_{\mathrm{A}}=V_{\mathrm{B}}=V_{\mathrm{C}}=V_{\mathrm{D}}=9.4 \mathrm{~V}$ confirms the parallel mode of LED load. When $V_{\mathrm{DC}}$ is in $15-24 \mathrm{~V}, E N \_2=E N \_3$ $=1, E N \_1=E N \_4=0, \mathrm{~S} 1, \mathrm{~S} 3, \mathrm{~S} 5, \mathrm{~S} 8$ are turned on to control LED_1 connects LED_2 in series, LED_3 connects LED_4 in series, and these two LED branches are connected in parallel. $V_{\mathrm{A}}=V_{\mathrm{C}}=19.7 \mathrm{~V}$ and $V_{\mathrm{B}}=V_{\mathrm{D}}=9.4 \mathrm{~V}$ confirms the hybrid mode of LED load. While $V_{\mathrm{DC}}$ is in $48-60 \mathrm{~V}, E N_{-} 1$ 
Table I Tested results of control vector, state of switches, connecting mode of LED segments, and voltage drop on each LED segment in the range of $15-60 \mathrm{~V}$ for $V_{\mathrm{DC}}$

\begin{tabular}{c|c|c|c}
\hline$V_{\mathrm{DC}}(\mathrm{V})$ & $15 \sim 24$ & $24 \sim 48$ & $48 \sim 60$ \\
\hline & $E N \_1=1$ & $E N \_1=0$ & $E N \_1=0$ \\
control signals & $E N \_2=1$ & $E N \_2=1$ & $E N \_2=0$ \\
& $E N \_3=0$ & $E N \_3=1$ & $E N \_3=1$ \\
& $E N \_4=0$ & $E N \_4=0$ & $E N \_4=1$ \\
\hline on-state & $\mathrm{S} 4, \mathrm{~S} 5, \mathrm{~S} 6, \mathrm{~S} 7$, & \multirow{2}{*}{$\mathrm{S} 1, \mathrm{~S} 3, \mathrm{~S} 5, \mathrm{~S} 8$} & $\mathrm{~S} 1, \mathrm{~S} 2, \mathrm{~S} 3$ \\
switches & $\mathrm{S} 8, \mathrm{~S} 9$ & $\mathrm{~S} 2, \mathrm{~S} 4, \mathrm{~S} 6, \mathrm{~S} 7$, & $\mathrm{S} 4, \mathrm{~S} 5, \mathrm{~S} 6, \mathrm{~S} 7$, \\
off-state & $\mathrm{S} 1, \mathrm{~S} 2, \mathrm{~S} 3$ & $\mathrm{~S} 9$ & $\mathrm{~S} 8, \mathrm{~S} 9$ \\
switches & & hybrid mode & series mode \\
\hline LED segments & \multirow{2}{*}{ parallel mode } & $V_{\mathrm{A}}=19.7$ & $V_{\mathrm{A}}=38.7$ \\
connecting & $V_{\mathrm{A}}=9.4$ & $V_{\mathrm{B}}=9.4$ & $V_{\mathrm{B}}=28.7$ \\
& $V_{\mathrm{B}}=9.4$ & $V_{\mathrm{C}}=19.7$ & $V_{\mathrm{C}}=19.7$ \\
voltage on LED & $V_{\mathrm{C}}=9.4$ & $V_{\mathrm{D}}=9.4$ & $V_{\mathrm{D}}=9.4$ \\
segments (V) & $V_{\mathrm{D}}=9.4$ & \multicolumn{2}{c}{}
\end{tabular}

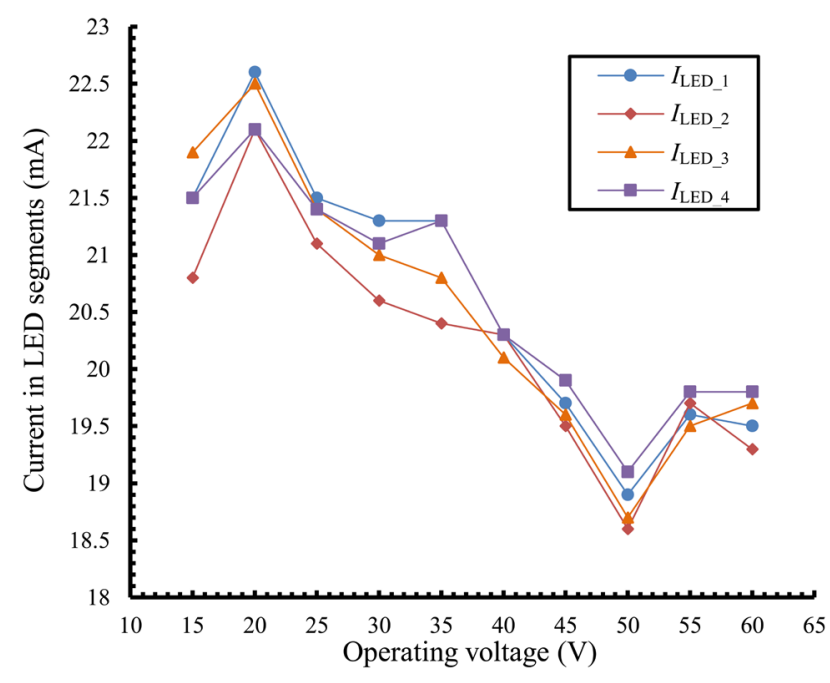

Fig. 3 Current in each LED segment obtained by varying $V_{D C}$.

$=E N \_2=0, E N \_3=E N \_4=1, \mathrm{~S} 1, \mathrm{~S} 2, \mathrm{~S} 3$ are turned on to control four LED segments connected in series. $V_{\mathrm{A}}=38.7 \mathrm{~V}$, $V_{\mathrm{B}}=28.7 \mathrm{~V}, V_{\mathrm{C}}=19.7 \mathrm{~V}$ and $V_{\mathrm{D}}=9.4 \mathrm{~V}$ confirms the series mode of LED load.

Fig. 3 indicates that tested current in each LED segment is stable at about $20 \mathrm{~mA}$ during $V_{\mathrm{DC}}$ in $15-60 \mathrm{~V}$. The voltage regulation rate for $I_{\text {LED } 1}, I_{\text {LED } 2}, I_{\text {LED } 3}$ and $I_{\text {LED } 4}$ is $66.7 \mu \mathrm{A} / \mathrm{V}, 82.2 \mu \mathrm{A} / \mathrm{V}, 77.8 \mu \mathrm{A} / \mathrm{V}, 84.4 \mu \mathrm{A} / \mathrm{V}$, respectively. As shown in Fig. 3, current in each LED segment decreases obviously when the input DC voltage is slightly greater than $48 \mathrm{~V}$. The reason is as following: When the input DC voltage is slightly greater than $48 \mathrm{~V}$, the connection mode of LED segments changes from hybrid mode to series mode. The voltage drop on LED load increases dramatically, resulting in a sharp decrease of voltage drop on the linear current block.

In Fig. 4, for the conventional constant current driving scheme which contains a CRD and 12 LEDs connected in series, when $V_{\mathrm{DC}}<30 \mathrm{~V}$, the LED power is about zero because the voltage drop on each LED is lower than its on voltage. LEDs do not reach their rate power until $V_{\mathrm{DC}}>$ $40 \mathrm{~V}$. For the proposed linear current driving scheme, in which the connection of load branches adaptively changes from parallel to hybrid, and finally to series as the operating voltage changes from low to high, the LED power is nearly invariable in the whole range of $V_{\mathrm{DC}}(15-60 \mathrm{~V})$, because the

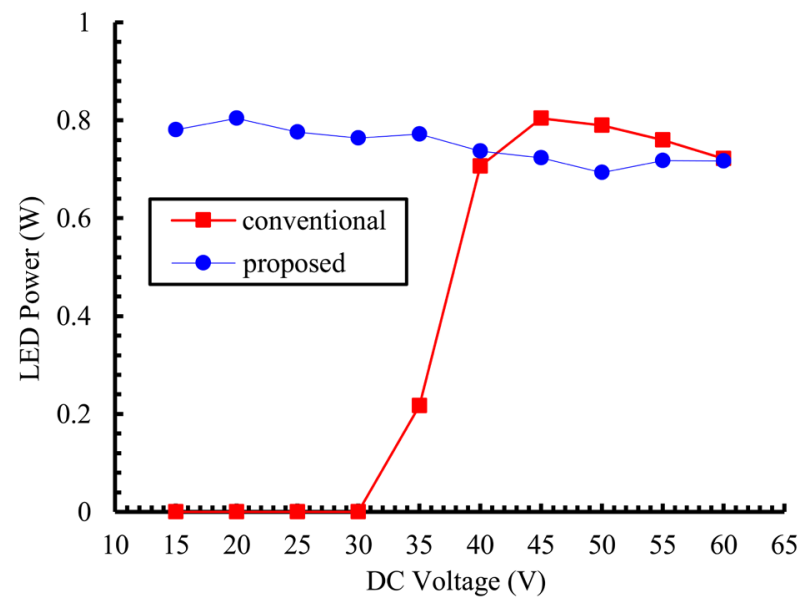

Fig. 4 Tested result of LED power of conventional constant current driving scheme and proposed linear current driving scheme.

current in each LED segment is constant, as shown in Fig. 3 in the whole range of $V_{\mathrm{DC}}$.

\section{Conclusion}

A novel linear current module adaptive to wide operating voltage range, combining the advantages of switch mode and linear mode current sources, is proposed. A switch array is employed to adaptively convert the series/hybrid/parallel connection mode of load segments according to the change of DC operating voltage. The proposed linear current module can make sure that the current in each load segment and the total power of load is constant, respectively, during wide operating voltage range. A four-segment LED (3 LEDs connect in series in each segment) driver is used to experimentally verify the proposed linear current module. During $15-60 \mathrm{~V}$ of operating voltage range, the current in each LED segment is stable around $20 \mathrm{~mA}$, and the total power of all four LED segments is nearly invariable $(0.693 \sim 0.804 \mathrm{~W})$. Compared with the conventional linear current LED driving scheme which contains a CRD and 12 LEDs connected in series, the operating voltage range of the proposed module is obviously wider.

\section{Acknowledgments}

This work was funded by Open Foundation of Engineering Research Center of Reliability of Semiconductor Power Devices of the Ministry of Education under grant 010201.

\section{References}

[1] P.J. Baxandall and E.W. Shallow: "Constant-current source with unusually high internal resistance and good temperature stability," Electron. Lett. 2 (1966) 351 (DOI: 10.1049/el:19660296).

[2] G.F. Walker: "Applications of a DC constant-current source," IEEE Spectr. 7 (1970) 47 (DOI: 10.1109/MSPEC.1970.5213555).

[3] F. You, et al.: "Am improved tail current source for low voltage applications," IEEE J. Solid-State Circuits 32 (1997) 1173 (DOI: 10.1109/4.604073).

[4] Y. Chuang and Y. Ke: "A novel high-efficiency battery charger with a buck zero-voltage-switching resonant converter," IEEE Trans. Energy Convers. 22 (2007) 848 (DOI: 10.1109/TEC.2006.882416).

[5] P. Annus, et al.: "Excitation current source for bioimpedance mea- 
surement applications: analysis and design,” IMTC (2008) 848 (DOI: 10.1109/IMTC.2008.4547155).

[6] Z. Yinggang, et al.: "Research on large power computer controlled constant current power source technologies," ICEMI (2009) 2-591 (DOI: 10.1109/ICEMI.2009.5274502).

[7] R.L. Nagaraj and D. Yagain: "Design and implementation of a linear transconductance amplifier with a digitally controlled current source," ICETET (2011) 274 (DOI: 10.1109/ICETET.2011.24).

[8] S.W. Han: "Research and design of LED driver power supply," M. S. Dissertation, Donghua University, Nanjing (2014).

[9] J. Ma, et al.: "Analysis on application of a current-source based DFIG wind generator model," CSEE Journal of Power and Energy Systems 4 (2018) 352 (DOI: 10.17775/CSEEJPES.2018.00060).

[10] Z. Dong, et al.: "Circuit theoretic considerations of LED driving: voltage-source versus current-source driving," IEEE Trans. Power Electron. 34 (2019) 4689 (DOI: 10.1109/TPEL.2018.2861914).

[11] J.-M. Routoure, et al.: "A low-noise and quasi-ideal DC current source dedicated to four-probe low-frequency noise measurements," IEEE Trans. Instrum. Meas. 69 (2020) 194 (DOI: 10.1109/TIM. 2019.2890891).

[12] R.H. Derksen: "Novel switched current source for increasing output signal edge steepness of current switches without generating large overshoot," IEEE J. Solid-State Circuits 30 (1995) 612 (DOI: 10.1109/4.384179).

[13] R. Beiranvand, et al.: "Designing an adjustable wide range regulated current source," IEEE Trans. Power Electron. 25 (2010) 197 (DOI: 10.1109/TPEL.2009.2027237).

[14] S.S. Lourdusami and R. Vairamani: "Analysis, design and experimentation of series-parallel LCC resonant converter for constant current source," IEICE Electron. Express 11 (2014) 1 (DOI: https://doi.org/10.1587/elex.11.20140711).

[15] S. Singh, et al:: "Improved power quality switched-mode power supply using buck-boost converter," IEEE Trans. Ind. Appl. 52 (2016) 5194 (DOI: 10.1109/TIA.2016.2600675).

[16] Y. Du: "Design of high precision and wide range current source based on microcontroller," IMCEC (2018) 2521 (DOI: 10.1109/IMCEC. 2018.8469519).

[17] J. He, et al.: "Quasi-selective harmonic elimination (Q-SHE) modulation-based DC current balancing method for parallel current source converters," IEEE Trans. Power Electron. 34 (2019) 7422 (DOI: 10.1109/TPEL.2018.2882463).

[18] H.A. Huynh, et al.: "Design and analysis of the DC-DC converter with a frequency hopping technique for EMI reduction," IEEE Trans. Compon. Packag. Manuf. Technol. 8 (2018) 546 (DOI: 10.1109/ TCPMT.2017.2788048).

[19] M.T. Tran, et al.: "EMI reduction and output ripple improvement of switching DC-DC converters with linear swept frequency modulation,” ICSICT (2018) 1 (DOI: 10.1109/ICSICT.2018.8565668).

[20] R. Sharms and T.R. Viswanathan: "Design of differential-voltagecontrolled current source," Proceedings of the Institution of Electrical Engineers 123 (1976) 319 (DOI: 10.1049/piee.1976.0073).

[21] M. Nakanishi, et al.: "Current dependence measurement of $1 \Omega$ standard resistors using a cryogenic current source with linear output," IEEE Trans. Instrum. Meas. 50 (2001) 255 (DOI: 10.1109/ 19.918115).

[22] K. Toyoda, et al.: "Proposal of a current regulative diode for power supply in sustained arc test," IEEE Trans. Plasma Sci. 34 (2006) 1967 (DOI: 10.1109/TPS.2006.881933).

[23] X. Wu, et al.: "A linear constant current LED driver without off-chip inductor and capacitor," NEWCAS (2015) 1 (DOI: 10.1109/ NEWCAS.2015.7181992).

[24] H. Martínez-García and A. Saberkari: "Linear-assisted DC/DC regulator-based current source for LED drivers," Electron. Lett. 52 (2016) 437 (DOI: 10.1049/el.2015.3437).

[25] C.X. Liu, et al.: "DC/DC converters combining switching power supply and linear power supply characteristics," Power Electron. 43 (2009) 24 (DOI: CNKI:SUN:DLDZ.0.2009-03-012).

[26] L. Hu, et al:: "Synchronous high step-down ratio non-isolated LED constant current driver based on improved Watkins-Johnson topology," IEEE Access 7 (2019) 18345 (DOI: 10.1109/ACCESS.2019. 2897057).
[27] A. Zu, et al:: "Analysis and design of a multi-channel constant current LED driver based on DC current bus distributed power system structure," IET Power Electron. 13 (2020) 627 (DOI: 10.1049/ iet-pel.2019.0203).

[28] J. Kim, et al.: "Isolated switch-mode current regulator with integrated two boost LED drivers," IEEE Trans. Ind. Electron. 61 (2014) 4649 (DOI: 10.1109/TIE.2013.2293700).

[29] J. Baek and S. Chae: "Off-line buck LED driver for series connected LED segments,” APEC (2017) 1506 (DOI: 10.1109/APEC.2017. 7930898).

[30] F.-K. Sun, et al.: "Research on thermal performance of AC-LED light engine driven by segmented linear constant current driver," ITOEC (2017) 220 (DOI: 10.1109/ITOEC.2017.8122415).

[31] J.G. Zhang: "Design of piecewise linear LED driver circuit," M.S. Dissertation, University of Electronic Science and Technology of China, Chengdu (2015). 